\title{
Non-equilibrium singlet-triplet Kondo effect in carbon nanotubes
}

\author{
J. PAASKE ${ }^{*}$, A. ROSCH ${ }^{2}$, P. WÖLFLE ${ }^{3}$, N. MASON ${ }^{4,5}$, C. M. MARCUS ${ }^{5}$ AND J. NYGÅRD ${ }^{1}$ \\ ${ }^{1}$ The Niels Bohr Institute \& The Nano-Science Center, University of Copenhagen, DK-2100 Copenhagen, Denmark \\ ${ }^{2}$ Institut für Theoretische Physik, Universität zu Köln, 50937 Köln, Germany \\ ${ }^{3}$ Institut für Theorie der Kondensierten Materie, Universität Karlsruhe, 76128 Karlsruhe, Germany \\ ${ }^{4}$ Department of Physics, University of Illinois at Urbana Champaign, Urbana, Illinois 61801-3080, USA \\ ${ }^{5}$ Department of Physics, Harvard University, Cambridge, Massachusetts 0213, USA \\ *e-mail: paaske@fys.ku.dk
}

T The Kondo effect is a many-body phenomenon arising due to conduction electrons scattering off a localized spin $^{1}$. Coherent spin-flip scattering off such a quantum impurity correlates the conduction electrons, and at low temperature this leads to a zero-bias conductance anomaly ${ }^{2,3}$. This has become a common signature in bias spectroscopy of single-electron transistors, observed in GaAs quantum $\operatorname{dots}^{4-9}$ as well as in various single-molecule transistors ${ }^{10-15}$. Although the zero-bias Kondo effect is well established, the extent to which Kondo correlations persist in non-equilibrium situations where inelastic processes induce decoherence remains uncertain. Here we report on a pronounced conductance peak observed at finite bias voltage in a carbon-nanotube quantum dot in the spin-singlet ground state. We explain this finite-bias conductance anomaly by a non-equilibrium Kondo effect involving excitations into a spin-triplet state. Excellent agreement between calculated and measured nonlinear conductance is obtained, thus strongly supporting the correlated nature of this non-equilibrium resonance.

For quantum dots accommodating an odd number of electrons, a suppression of charge fluctuations in the Coulomb-blockade regime leads to a local spin-1/2 degree of freedom and, when temperature is lowered through a characteristic Kondo temperature $T_{\mathrm{K}}$, the Kondo effect shows up as a zero-bias peak in the differential conductance. In a dot with an even number of electrons, the two electrons residing in the highest occupied level may either form a singlet or promote one electron to the next level to form a triplet, depending on the relative magnitude of the level splitting $\delta$ and the ferromagnetic intradot exchange energy $J$. For $J>\delta$, the triplet state prevails and gives rise to a zero-bias Kondo peak $^{7,8}$, but when $\delta>J$ the singlet state is lower in energy and no Kondo effect is expected in the linear conductance. Nevertheless, spin-flip tunnelling becomes viable when the applied bias is large enough to induce transitions from singlet to triplet states. Such interlead exchange-tunnelling may give rise to Kondo correlations

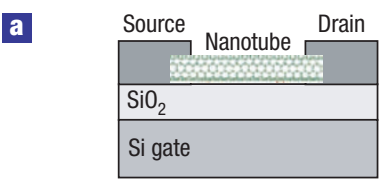

b

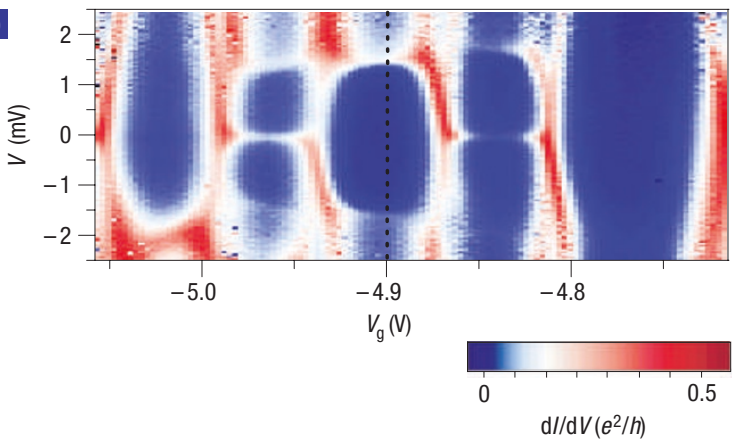

G

\begin{tabular}{|c|c|c|c|c|}
\hline$N=0$ & $N=1$ & $N=2$ & $N=3$ & $N=4$ \\
\hline & & & & 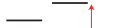 \\
\hline - & 平 & $A_{ \pm}$ & $A_{t} \uparrow$ & $\mathcal{A}_{\downarrow} \mathcal{A}_{\downarrow} \delta$ \\
\hline$S=0$ & $S=1 / 2$ & $S=0$ & $S=1 / 2$ & $S=0$ \\
\hline
\end{tabular}

Figure 1 Experimental setup and shell-filling scheme for a single-wall carbon nanotube. a, Schematic diagram of the nanotube device, comprising a single-wall carbon nanotube grown by chemical vapour deposition on $\mathrm{SiO}_{2}$ substrate and contacted by $\mathrm{Cr} / \mathrm{Au}$ source and drain electrodes, spaced $250 \mathrm{~nm}$ apart. Highly doped silicon below the $\mathrm{Si}_{2}$ cap layer acted as a back-gate electrode. Room-temperature measurements of conductance as a function of back-gate voltage, $V_{g}$, indicate that the conducting nanotube is metallic with a small gap outside the region considered here (see Supplementary Information, Section S1). b. Density plot of $\mathrm{d} / / \mathrm{d} V$ as a function of $V$ and $V_{\mathrm{g}}$ at $T_{\mathrm{el}}=81 \mathrm{mK}$. c, Diagram illustrating the corresponding level filling scheme with $N$ defined as the total number of electrons modulo 4 . 


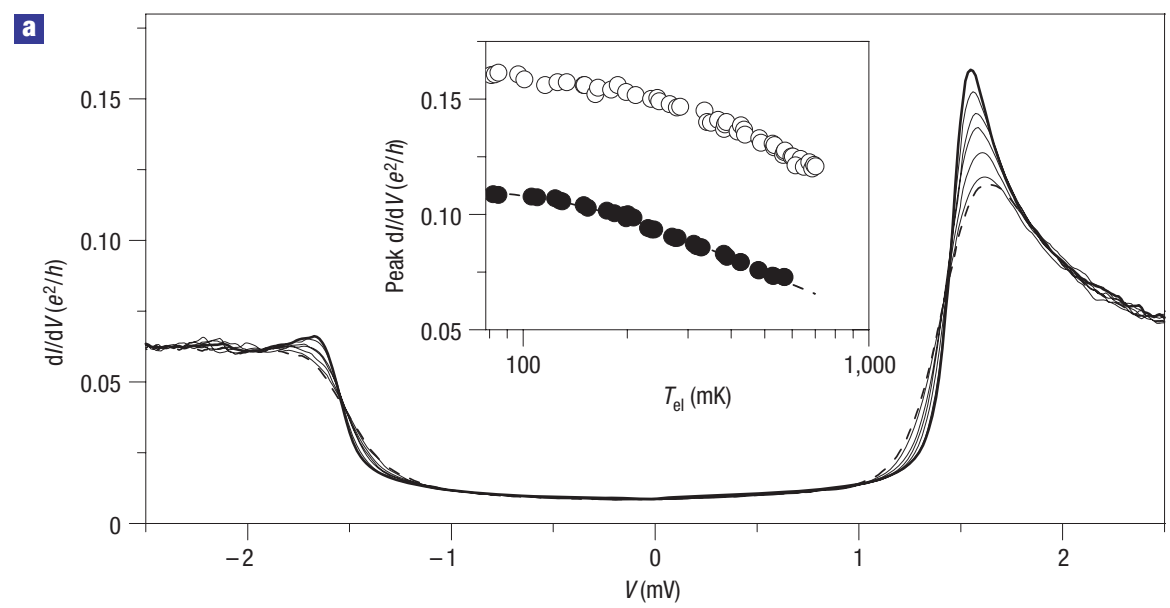

b

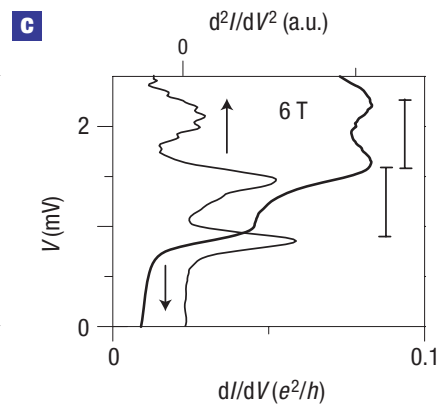

Figure 2 Temperature and magnetic-field dependence of the finite-bias resonance. $\mathrm{a}, \mathrm{d} / \mathrm{d} V$ as a function of $V$, at $V_{g}=-4.90 \mathrm{~V}$ (dashed cross-section in Fig. $1 \mathrm{~b}$ ), taken at $T_{\text {el }}=81$ (thick line), 199, 335, 388, 488, 614, 687 (dashed line) mK. Inset: temperature dependence of the finite-bias conductance peak in a (open circles) and the neighbouring zero-bias Kondo resonance (filled circles) at $V_{g}=-4.96 \mathrm{~V}$ (see Fig. 1a). The open circles are consistent with the saturation of a log-enhanced finite-bias Kondo peak as $T$ becomes smaller than the spin-relaxation rate $\Gamma \approx 250 \mathrm{mK}$, determined from the parameters in Fig. 4 . The filled circles follow the numerical renormalization group interpolation formula $a\left(1+\left(2^{1 / 0.22}-1\right)\left(T / T_{\mathrm{K}}\right)^{2}\right)^{-0.22}$ with $a=0.11$ and $T_{\mathrm{K}}=1.0 \mathrm{~K}$ (dashed line). $\mathbf{b}, \mathrm{d} l / \mathrm{d} V$ versus $V$ and magnetic field $B$, at $V_{\mathrm{g}}=-4.90 \mathrm{~V}$. c, $\mathrm{d} / / \mathrm{d} V$ versus $V$ at $B=6 \mathrm{~T}$, corresponding to vertical cross-section (dashed) in $\mathbf{b}$. The $\mathrm{d}^{2} I / \mathrm{d} V^{2}$ trace (thin line) underlines the presence of three distinct peaks in $\mathrm{d} l / \mathrm{d} V$. The black bars each correspond to $\Delta V=g \mu_{\mathrm{B}} B / e$ with $g=2.0$ for nanotubes.

and concomitant conductance peaks near $V \sim \pm \delta / e$, where $e$ is the elementary charge. However, because the tunnelling involves excited states with a rather limited lifetime, the extent to which the coherence of such inelastic spin flips, and hence the extent to which the Kondo effect is maintained, remains unanswered.

The possibility of finite-bias conductance anomalies induced by the Kondo effect was discussed in 1966 by Appelbaum ${ }^{16}$, and a detailed account of the subsequent development of this problem can be found in ref. 17. In the context of a double-dot system, a qualitative description of a finite-bias Kondo-effect, treating decoherence and non-equilibrium effects in an incomplete way, is given in ref. 18. Finite-bias conductance peaks have already been observed in carbon nanotubes ${ }^{11,14,19}$ as well as in GaAs quantum $\operatorname{dots}^{8,9,20}$. However, owing to the lack of a quantitative theory for this non-equilibrium resonance, characterization of the phenomenon has not yet been possible. As pointed out in refs 17,21,22, a bias-induced population of the excited state (here the triplet), may change a simple finite-bias cotunnelling step into a cusp in the nonlinear conductance. Therefore, to quantify the strength of correlations involved in such a finite-bias conductance anomaly, a proper non-equilibrium treatment will be necessary. As we demonstrate below, the qualitative signature of Kondo correlations is a finite-bias conductance peak which is markedly sharper than the magnitude of the threshold bias.

We have examined a quantum dot based on a single-wall carbon nanotube (see Fig. 1a). Electron transport measurements of the two-terminal differential conductance were carried out in a cryostat with a base electron temperature of $T_{\mathrm{el}} \approx 80 \mathrm{mK}$ and a magnetic field perpendicular to the nanotube axis. The low-temperature characteristics of the device are seen from the density plot in Fig. $1 \mathrm{~b}$, showing $\mathrm{d} I / \mathrm{d} V$ as a function of source-drain voltage $V$ and gate voltage $V_{\mathrm{g}}$. The dominant blue regions of low conductance are caused by Coulomb blockade (CB), whereas the sloping white and red lines are edges of the $\mathrm{CB}$ diamonds, where the blockade is overcome by the finite source-drain bias. Moreover, white and red horizontal ridges of high conductance around zero bias are seen. These ridges occur in an alternating manner, for every second electron added to the nanotube dot. These are the Kondo resonances induced by the finite electron spin $S=1 / 2$ existing for an odd number $N$ of electrons where an unpaired electron is localized on the tube. The zero-bias resonances are absent for the other regions (with even $N$ ) where the ground-state spin is $S=0$. Over most of the measured gate-voltage range (not all shown), the diamond plot shows a clear four-electron periodicity, consistent with the consecutive filling of two non-degenerate sub-bands, corresponding to the two different sublattices of the rolled-up graphene sheet, within each shell ${ }^{19}$. This shell-filling scheme is illustrated in Fig. 1c.

We observe inelastic cotunnelling features for all even $N$ at $e V \sim \pm \Delta$ for a filled shell and $e V \sim \pm \delta$ for a half-filled shell. Reading off the addition, and excitation energies throughout the quartet near $V_{\mathrm{g}}=-4.90 \mathrm{~V}$, shown in Fig. 1b, we can estimate the 

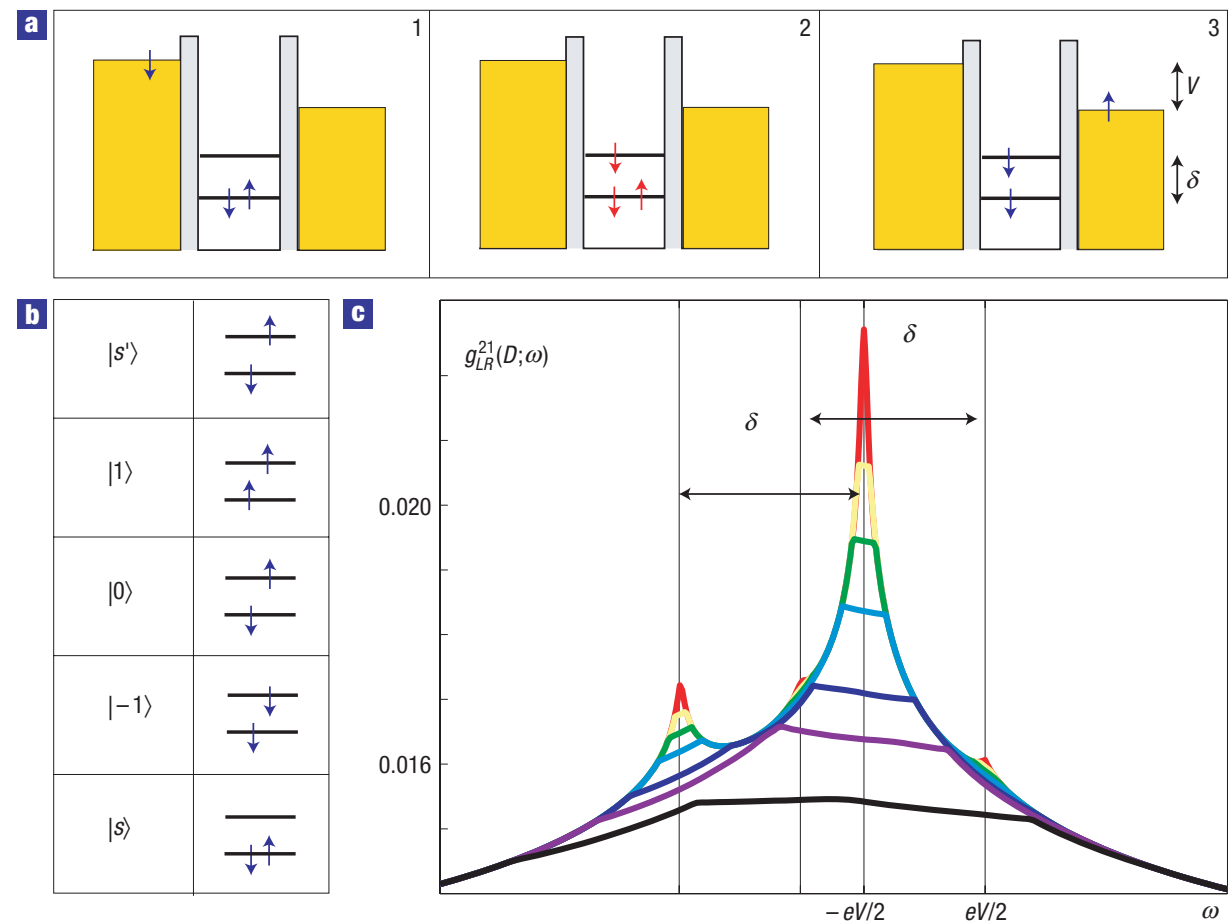

Figure 3 Schematic diagram of the inelastic spin-exchange underlying the Kondo effect. a, lllustration of the cotunnelling mechanism giving rise to the effective exchange interaction in the Kondo model. Note that the virtual intermediate state (red spins) in panel 2 is suppressed by a large energy denominator of the order of the electrostatic charging energy of the nanotube. $\mathbf{b}$, Schematic diagram of the five different low-energy states retained in the effective Kondo model: $|s\rangle$ is the singlet ground state, $|m\rangle(m=1,2,3)$ are the triplet components with excitation energy $\delta-J \approx \delta$ and $\left|s^{\prime}\right\rangle$ is the corresponding singlet with excitation energy $\delta$. Applying a magnetic field causes the triplet to split up as seen in Fig. 2b,c. c, Renormalized dimensionless exchange-coupling versus incoming conduction electron energy $\omega$, measuring the amplitude for exchange-tunnelling from right to left lead while de-exciting an electron on the nanotube from orbital 2 to 1 . The different colours encode the different stages of the $\mathrm{RG}$ flow as the bandwidth is reduced from $D_{0}$ (black) to zero (red). Parameters as in Fig. 4.

relevant energy scales within a constant interaction model ${ }^{19,23,24}$. We deduce a charging energy $E_{\mathrm{C}} \approx 3.0 \mathrm{meV}$, a level spacing $\Delta \approx 4.6 \mathrm{meV}$, a sub-band mismatch $\delta \approx 1.5 \mathrm{meV}$, and rather weak intradot exchange, and intra-orbital Coulomb energies $J, \mathrm{~d} U<0.05 \delta$. The fact that $\delta>J$ is consistent with a singlet ground state for a half-filled shell, involving only the lower sub-band (orbital), together with a triplet at excitation energy $\delta-J$ and another singlet at energy $\delta$. It should also be noted that the regions with odd $N$ and doublet ground state have an inelastic resonance at an energy close to $\delta$. We ascribe these to (possibly Kondo-enhanced) transitions exciting the valence electron from orbital 1 to orbital 2, but we shall not investigate these resonances in detail. Focusing on the half-filled shell, that is $N=2$, Fig. 2a shows the measured line shape, $\mathrm{d} I / \mathrm{d} V$ versus $V$, at $V_{\mathrm{g}}=-4.90 \mathrm{~V}$ and for $T_{\mathrm{el}}$ ranging from 81 to $687 \mathrm{mK}$. The conductance is highly asymmetric in bias voltage and exhibits a pronounced peak near $V \sim \delta / e$ which increases markedly when lowering the temperature (see Fig. 2a, inset).

For $N=2$, we model the nanotube quantum dot by a two-orbital Anderson impurity occupied by two electrons coupled to the leads by means of four different tunnelling amplitudes $t_{i \alpha}$, between orbitals $i=1,2$ and leads $\alpha=L, R$. In terms of the conduction electron density of states, $v_{\mathrm{F}}$, which we assume to be equal for the two leads, the tunnelling induces a level broadening $\Gamma_{i}=2 \pi v_{\mathrm{F}} \sum_{\alpha}\left|t_{i \alpha}\right|^{2}$. In the Kondo regime, $\Gamma_{i} \ll E_{\mathrm{C}}$, charge fluctuations are strongly suppressed and the Anderson model becomes equivalent to a Kondo model incorporating second-order superexchange (cotunnelling) processes, like that illustrated in Fig. 3a, in an effective exchange-tunnelling interaction (see Supplementary Information, Section S2). This effective model describes the possible transitions between the five lowest-lying two-electron states on the nanotube (see Fig. 3b). As $J \ll \delta$, we neglect $J$ and assume that the four excited states are all degenerate with excitation energy $\delta$. The next excited state is a singlet entirely within orbital 2 with excitation energy $2 \delta$ (see Supplementary Information, Section S2). Although this state is merely a factor of two higher in energy than the five lowest-lying states, we shall neglect this and all higher-lying states. As there are no spin-flip transitions connecting the ground-state singlet to the excited singlet at energy $2 \delta$, we expect this omission to have a minute influence on the conductance for $V<2 \delta$.

To deal with the logarithmic singularities arising in low-order perturbation theory (PT) within the Kondo model, it is convenient to use the so-called poor man's scaling method ${ }^{25}$. As we have demonstrated earlier ${ }^{26}$, this method can also be generalized to deal with non-degenerate spin states, such as a spin-1/2 in a magnetic field, as well as with non-equilibrium systems where the local spin degrees of freedom are out of thermal equilibrium with the conduction electrons in the leads. The present problem is complicated by the presence of the two finite energy scales, $\mathrm{eV}$ and $\delta$, causing the renormalization of the couplings to involve more than just scattering near the conduction-electron Fermi surfaces. To accommodate for this fact, we must allow for different renormalization at different energy scales, and the poor man's 

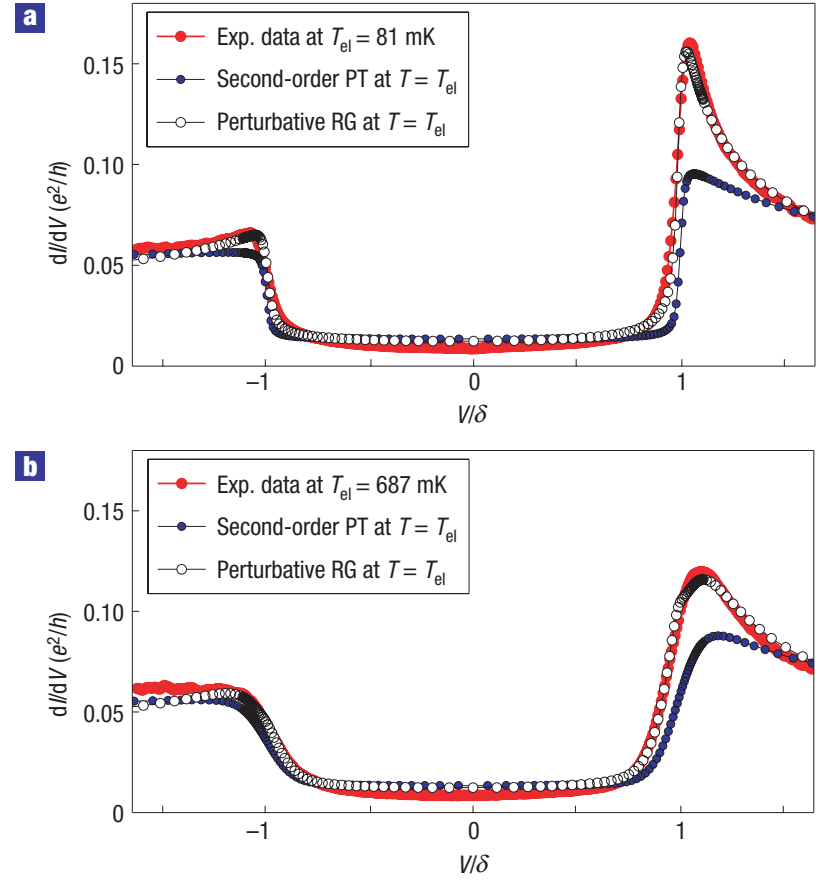

Figure 4 Fitting the nonlinear conductance by perturbative $\mathrm{RG}$ calculation. a, Experimental data (red circles) at the lowest temperature, fitted by the perturbative $R G$ calculation (white circles) with $\left\{t_{L 1}, t_{L 2}, t_{R 1}, t_{R 2}\right\}=$ $\{0.032,0.028,0.108,0.063\} \sqrt{E_{\mathrm{C}} / \nu_{\mathrm{F}}}$ and $T=T_{\mathrm{el}}=81 \mathrm{mK}$. We use $J=0$ and an arbitrary bandwidth, $D_{0}=1 \mathrm{eV}$, larger than all other energy scales. From the bias at which $\mathrm{d} / / \mathrm{d} V$ versus $V$ has maximum slope, we read off $\delta \approx 1.5 \mathrm{meV}$. The blue circles represent simple cotunnelling, that is, second-order PT with tunnelling amplitudes $t_{i \alpha}$ fitted to the data at $T=687 \mathrm{mK}$ see (b). b. Comparison with the data (red and white circles) at the highest temperatures, using the same parameters as in a, but with $T=T_{\mathrm{el}}=687 \mathrm{mK}$. The blue curve represents the best fit of second-order PT with $T=687 \mathrm{mK}$.

scaling method should therefore be generalized to produce a renormalization group (RG) flow of frequency-dependent coupling functions. The details of this method are presented in refs 26,27 and the specific RG equations for this problem are presented in the Supplementary Information, Section S3.

These coupled nonlinear RG equations do not lend themselves to analytical solution but may be solved numerically with relatively little effort. From this solution, we obtain renormalized coupling functions with logarithmic peaks at certain frequencies determined by $e V$ and $\delta$ (see, for example, Fig. 3c). As resonant spin flips only take place through the excited states at energy $\delta$, Korringa-like spin relaxation, through excitation of particle-hole pairs in the leads, partly degrades the coherence required for the Kondo effect ${ }^{28}$. Therefore, even at zero temperature, the couplings remain weak and increase roughly as $1 / \ln \left(\sqrt{T^{2}+\Gamma^{2}} / T_{\mathrm{K}}\right)$, with $\Gamma$ being the effective ( $V$-dependent) spin-relaxation rate. Using the parameters $t_{\alpha i}$ from the fit in Fig. 4, we calculate the Kondo temperature and the spin-relaxation rate and find that $T_{\mathrm{K}} \approx 2 \mathrm{mK}$ and $\Gamma \approx 250 \mathrm{mK}$ (see Supplementary Information, Sections S3-S5). This in turn implies a small parameter $1 / \ln \left(\delta / T_{\mathrm{K}}\right) \approx 0.1$, which justifies our perturbative approach. Notice that the smallness of $T_{\mathrm{K}}$ corresponds roughly to a mere $30 \%$ reduction of the total hybridization to orbital 1 as an electron is added to the nanotube. This tiny $T_{\mathrm{K}}$ does not show up directly in the $V$-dependence of the nonlinear conductance, for example, in the width of the peak, which is characterized instead by the excitation energy $\delta$ and the spin-relaxation rate $\Gamma$.

Having determined the renormalized coupling functions, the electrical current is finally calculated from the golden rule expression:

$$
\begin{aligned}
I= & \frac{2 \pi e}{\hbar} \int_{-\infty}^{\infty} \mathrm{d} \omega \sum_{\substack{\sigma, \sigma^{\prime}=\uparrow, \downarrow \\
\gamma, \gamma^{\prime}=s,-1,0,1, s^{\prime}}}\left|g_{R, \sigma^{\prime} ; L, \sigma}^{\gamma^{\prime}, \gamma}(\omega)\right|^{2} f\left(\omega-\mu_{L}\right) \\
& \times\left(1-f\left(\omega+\varepsilon_{\gamma}-\varepsilon_{\gamma^{\prime}}-\mu_{R}\right)\right) n_{\gamma}-(L \leftrightarrow R),
\end{aligned}
$$

where $\mu_{L / R}= \pm e V / 2$ denotes the two different chemical potentials, $\hbar$ is Planck's constant, $n_{\gamma}$ are the non-equilibrium occupation numbers of the five impurity states and $g_{R, \sigma^{\prime} ; L, \sigma}^{\gamma^{\prime} ; \gamma}(\omega)$ is the renormalized exchange-tunnelling amplitude for transferring a conduction electron from left to right lead and changing its spin from $\sigma$ to $\sigma^{\prime}$, while switching the impurity state from $\gamma$ to $\gamma^{\prime}$. Figure 4 shows a comparison of this calculation to the data, obtained by fitting the four tunnelling amplitudes $t_{i \alpha}$ determining the unrenormalized exchange couplings. Note that the measured conductance at $V=0$ and at $|V| \gg \delta$, together with the asymmetry between positive and negative bias, places strong constraints on these four amplitudes. The resulting fit is highly satisfactory, with only slight deviations at the highest voltages.

To quantify the importance of Kondo correlations, we also make a comparison with plain non-equilibrium cotunnelling. This unrenormalized second-order tunnelling mechanism clearly underestimates the conductance peak at positive bias, and most severely so at the lowest temperature. Qualitatively, simple non-equilibrium cotunnelling gives rise to a cusp in the conductance which is roughly as wide as the magnitude of the threshold bias itself. As is evident from the data and analysis presented here, non-equilibrium Kondo correlations instead produce a conductance anomaly which is sharper than the magnitude of the threshold-that is a proper finite-bias peak.

Received 18 November 2005; accepted 25 May 2006; published 1 July 2006.

References

1. Hewson, A. C. The Kondo Problem to Heavy Fermions (Cambridge Univ. Press, Cambridge, 1993).

2. Glazman, L. \& Raikh, M. Resonant Kondo transparency of a barrier with quasilocal impurity states. JETP Lett. 47, 452-455 (1988).

3. Ng, T. \& Lee, P. A. On-site Coulomb repulsion and resonant tunnelling. Phys. Rev. Lett. 61, 1768-1771 (1988).

4. Goldhaber-Gordon, D. et al. Kondo effect in a single-electron transistor. Nature 391, 156-159 (1998).

5. Cronenwett, S. M., Oosterkamp, T. H. \& Kouwenhoven, L. P. A tunable Kondo effect in quantum dots. Science 281, 540-544 (1998).

6. van der Wiel, W. G. et al. The Kondo effect in the unitary limit. Science 289, 2105-2108 (2000).

7. Sasaki, S. et al. Kondo effect in an integer-spin quantum dot. Nature 405, 764-767 (2000).

8. Kogan, A., Granger, G., Kastner, M. A. \& Goldhaber-Gordon, D. Singlet-triplet transition in a single-electron transistor at zero magnetic field. Phys. Rev. B 67, 113309 (2003).

9. Zumbühl, D. M., Marcus, C. M., Hanson, M. P. \& Gossard, A. C. Cotunnelling spectroscopy in few-electron quantum dots. Phys. Rev. Lett. 93, 256801 (2004).

10. Park, J. et al. Coulomb blockade and the Kondo effect in single-atom transistors. Nature 417, 722-725 (2002).

11. Nygård, J., Cobden, D. H. \& Lindelof, P. E. Kondo physics in carbon nanotubes. Nature 408, 342-346 (2000)

12. Liang, W., Shores, M. P., Bockrath, M., Long, J. R. \& Park, H. Kondo resonance in a single-molecule transistor. Nature 417, 725-729 (2002).

13. Yu, L. H. et al. Inelastic electron tunnelling via molecular vibrations in single molecule transistors. Phys. Rev. Lett. 93, 266802 (2004).

14. Babić, B., Kontos, T. \& Schönenberger, C. Kondo effect in carbon nanotubes at half filling Phys. Rev. B 70, 235419 (2004).

15. Jarillo-Herrero, P. et al. Orbital Kondo effect in carbon nanotubes. Nature 434, 484-488 (2005).

16. Appelbaum, J. 's-d' exchange model of zero-bias tunnelling anomalies. Phys. Rev. Lett. 17, 91-95 (1966).

17. Paaske, J., Rosch, A. \& Wölfle, P. Nonequilibrium transport through a Kondo dot in a magnetic field: perturbation theory. Phys. Rev. B 69, 155330 (2004).

18. Kiselev, M. N., Kikoin, K. \& Molenkamp, L. W. Resonance kondo tunnelling through a double quantum dot at finite bias. Phys. Rev. B 68, 155323 (2003).

19. Liang, W., Bockrath, M. \& Park, H. Shell filling and exchange coupling in metallic single-walled carbon nanotubes. Phys. Rev. Lett. 88, 126801 (2002).

20. Jeong, H., Chang, A. M. \& Melloch, M. R. The Kondo effect in an artificial quantum dot molecule. Science 293, 2221-2223 (2001).

21. Wegewijs, M. R. \& Nazarov, Yu. V. Inelastic co-tunnelling through an excited state of a quantum dot. Preprint at $<$ http://arxiv.org/abs/cond-mat/0103579> (2001). 


\section{LETTERS}

22. Golovach, V. N. \& Loss, D. Transport through a double quantum dot in the sequential tunnelling and cotunnelling regimes. Phys. Rev. B 69, 245327 (2004).

23. Oreg, Y., Byczuk, K. \& Halperin, B. I. Spin configurations of a carbon nanotube in a nonuniform external potential. Phys. Rev. Lett. 85, 365-368 (2000).

24. Sapmaz, S. et al. Electronic excitation spectrum of metallic carbon nanotubes. Phys. Rev. B 71, 153402 (2005).

25. Anderson, P. W. A poor man's derivation of scaling laws for the Kondo problem. J. Phys. C 3, 2436-2441 (1966).

26. Rosch, A., Paaske, J., Kroha, J. \& Wölfle, P. Nonequilibrium transport through a Kondo dot in a magnetic field: perturbation theory and poor man's scaling. Phys. Rev. Lett. 90, 076804 (2003).

27. Rosch, A., Paaske, J., Kroha, J. \& Wölfle, P. The Kondo effect in non-equilibrium quantum dots: perturbative renormalization group. J. Phys. Soc. Japan 74, 118-126 (2005).

28. Paaske, J., Rosch, A., Kroha, J. \& Wölfle, P. Nonequilibrium transport through a Kondo dot: decoherence effects. Phys. Rev. B 70, 155301 (2004)

\section{Acknowledgements}

We thank L. DiCarlo and W. F. Koehl for experimental contributions and D. H. Cobden and V. Körting for useful discussions. This research was supported by the Center for Functional Nanostructures of the DFG (J.P., P.W.), the European Commission through project FP6-003673 CANEL of the IST Priority (J.P.), ARO/ARDA (DAAD19-02-1-0039), NSF-NIRT (EIA-0210736) (N.M., C.M.M.) and the Danish Technical Research Council (J.N.).

Correspondence and requests for materials should be addressed to J.P.

Supplementary Information accompanies this paper on www.nature.com/naturephysics.

\section{Competing financial interests}

The authors declare that they have no competing financial interests.

Reprints and permission information is available online at http://npg.nature.com/reprintsandpermissions/ 